\title{
Calcificaciones cerebrales: enfermedad 0 síndrome de Fahr
}

\section{Brain calcifications: Fahr's disease or syndrome}

\author{
Carlos Alberto Dávila-Hernández' \\ Galo Franks Bendezú-Ramos ${ }^{2}$ \\ Marianella Torres-Luján ${ }^{2}$ \\ Julissa Enimia del Rosario Cárdenas-Trejo 3 \\ Diana Esther Picoy-Romero ${ }^{4}$
}

Dávila-Hernández C, Bendezú-Ramos G,Torres-Luján M, Cárdenas-Trejo J, Picoy-Romero D. Calcificaciones cerebrales: enfermedad o síndrome de Fahr. Rev Soc Peru Med Interna. 202 I;34(I): I2-I4. https://doi.org/10.36393/spmi.v34i1.579

\section{RESUMEN}

Paciente mujer de 58 años de edad, fue traída por presentar miclonías, convulsiones tónico-clónicas, con antecedentes de hipertensión, diabetes mellitus, hipotiroidismo, secuela de infarto cerebral. A la exploración clínica se le halló soporosa, pupilas isocóricas, hiporreactivas, hemiparesia derecha. El estudio tomográfico cerebral sin contraste mostró calcificaciones cerebrales y cerebelosas. Se discute sobre los conceptos de enfermedad o síndrome de Fahr.

Palabras claves: enfermedad de Fahr, síndrome de Fahr, calcificaciones cerebrales

\section{ABSTRACT}

A 58-year-old female patient was brought in for myclonies, tonic-clonic seizures, with a history of hypertension, diabetes mellitus, hypothyroidism, cerebral infarction sequelae. Clinical examination was found soporous, isochoric pupils, hyporeactive, right hemiparesis. The no-contrast brain tomographic study showed cerebral and cerebellar calcifications. It is discussed about the concepts of Fahr disease or syndrome.

Keywords: Fahr disease, Fahr syndrome, brain calcifications

I Médico internista.Hospital IVAHM-EsSalud,Ica.Facultad de Medicina Humana Daniel Alcides Carrión, Universidad Nacional San Luis Gonzaga de Ica, Perú

2 Médico residente de medicina interna. Hospital IVAHM-EsSalud, Ica, Perú.

3 Médico residente de radiología. Hospital IV AHM - EsSalud, Ica, Perú.

4 Médico residente de patología clínica. Hospital IV AHM - EsSalud, Ica, Perú.

\section{INTRODUCCIÓN}

En el año 1 930, Karl Theodor Fahr patólogo alemán, realizó su primera descripción titulada "calcificaciones idiopáticas de los vasos cerebrales", de donde se originó el epónimo de enfermedad de Fahr, en un paciente con demencia de larga evolución, que presentaba calcificaciones bilaterales, alrededor de los ventrículos laterales y ganglios de la base, en ausencia de lesión arteriosclerótica. ${ }^{1,2}$ Las pequeñas calcificaciones, "fisiológicas", se localizan particularmente a nivel de los globos pálidos y aumentan su prevalencia con la edad, cuando se presentan en sujetos menores de 40 años y comprometen simultáneamente pálido, putamen, núcleo dentado del cerebelo y, eventualmente, la sustancia blanca hemisférica, y se las considera patológicas. ${ }^{3}$ Se le reporta ocasionalmente. $^{4}$

La enfermedad de Fahr y el síndrome de Fahr son dos afecciones poco comunes que se caracterizan por la calcificación en ciertas áreas del cerebro que resultan en manifestaciones neurológicas y psiquiátricas. Las 
calcificaciones son depósitos anómalos de calcio, y otros minerales como magnesio, zinc, hierro y aluminio. La presencia de estas calcificaciones ha llevado al empleo de múltiples denominaciones para esta misma patología. ${ }^{5}$ Ambas afecciones pueden parecerse entre sí, pero existen diferencias claras con respecto a la etiología, la ubicación de las lesiones, el pronóstico y el tratamiento. Por lo tanto, es importante conocer las similitudes y diferencias de ambas afecciones para que se pueda realizar el diagnóstico y tratamiento adecuado, de este trastorno neurológico de baja prevalencia en nuestro medio.

\section{PRESENTACIÓN DEL CASO}

Paciente mujer de 58 años de edad, que ingresó al servicio de emergencia por presentar trastorno del sensorio ("mirada fija y no contesta al llamado") y antes de esto presentó como "sacudidas en todo el cuerpo" sin relajación de esfínteres, sin sialorrea y sin retroversión ocular. Durante todo el trayecto al hospital, la hija manifestó "venido convulsionando su brazo y pierna izquierda". Niegan traumatismos recientes. Niegan ingestión de sustancias desconocidas.

Antecedentes de hipertensión arterial, toma losartán 50 mg cada 12 horas; diabetes mellitus, recibe insulina NPH 30 UI SC en la mañana y en la noche; hipotiroidismo, toma levotiroxina $100 \mathrm{mcg}$ cada 24 horas; todos ellos con control irregular); infartos cerebrales hace 6 años y 2 años, respectivamente.

Al examen físico: PA 90/60 mm Hg; FC 108 latidos/ min; FR $24 \mathrm{resp} / \mathrm{min}$. Edema de miebros inferiores $+/ 3+$. Pulmones: murmullo respiratorio disminuido en la base del hemitórax derech. Cardiovascular: ruidos cardiacos rítmicos, taquicárdicos, de baja intensidad. Abdomen sin alteraciones. SN: soporosa, pupilas isocóricas e hiporreactivas; disminución de la fuerza muscular en el hemicuerpo derecho, no signos meníngeos; durante el examen, presentó movimientos tónico-clónicos generalizados que cedieron con la aplicación endovenosa de diazepam $10 \mathrm{mg}$.

Se inició tratamiento anticonvulsivo y por posible neumonía aspirativa.

Exámenes auxiliares: hemograma: leucocitos $15,830 / \mathrm{mm}^{3}$; $\mathrm{Hb} 7,8 \mathrm{~g} / \mathrm{dl}$; plaquetas $250 / \mathrm{mm}^{3}$; abastonados $6 \%$; VCM 84,5f; HCM 28,8 pgr. PCR 20,79 mg/dl, glucosa $301 \mathrm{mg} / \mathrm{dl}$, urea $79,2 \mathrm{mg} / \mathrm{dl}$, creatinina $0,65 \mathrm{mg} / \mathrm{dl}$, sodio $145 \mathrm{mmol} / \mathrm{L}$,
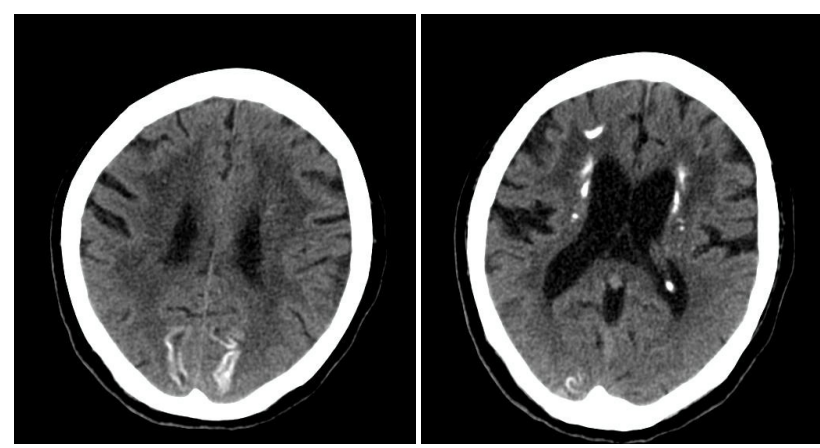

potasio 4,1 mmol/L. Sedimento urinario: leucocitos 30-35/ campo, hematíes 10-15/campo, células epiteliales escasas, levaduras: ++ .

Radiografía de tórax: tenue opacidad basal derecha. Tomografía cerebral sin contraste: calcificaciones cerebrales y cerebelosas, signos en relación a envejecimiento cerebral; angioesclerosis de carótidas internas (Figuras 1-6).

Se amplió historia clínica con otro familiar, otra hija, quien refirió que la paciente había recibido atención de manera esporádica por psiquiatría con el diagnóstico de trastorno mixto de ansiedad y depresión, recibiendo sertralina 50 $\mathrm{mg}$ vía oral por la mañana y clonazepam $2 \mathrm{mg}$ vía oral por las noches, mostrando parcialmente mejoría. La paciente pasa a sala de observación para completar los estudios pero falleció.
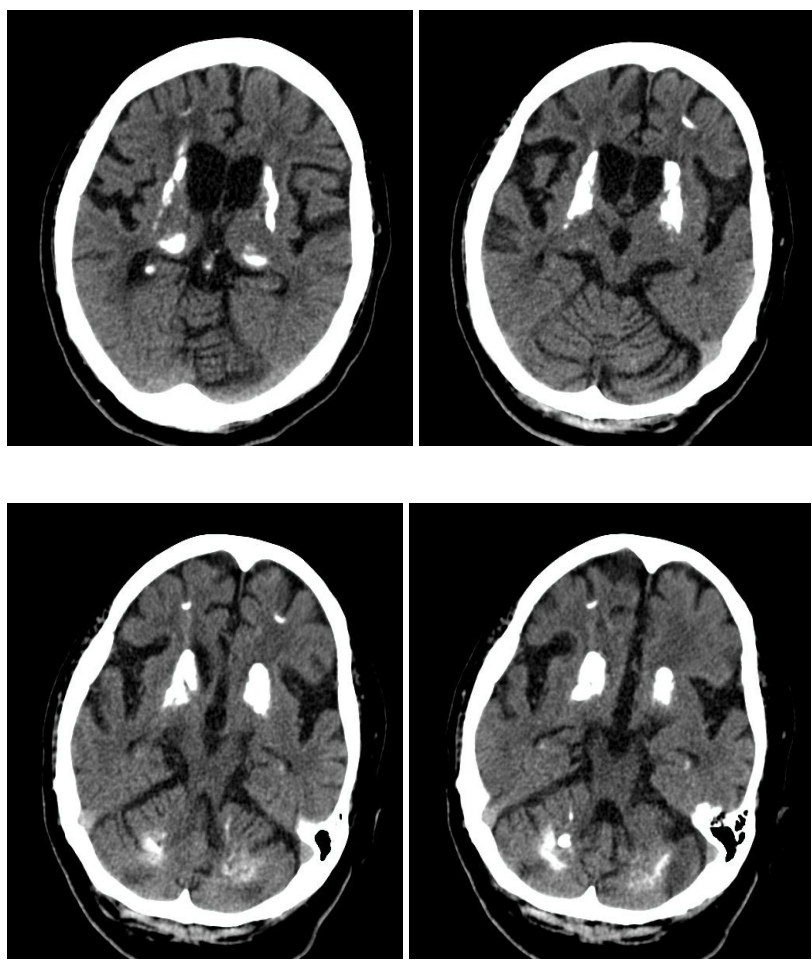

\section{DISCUSIÓN}

La enfermedad de Fahr es una entidad con transmisión hereditaria de forma autosómica dominante, localizada en el brazo corto del cromosoma 14, caracterizada por calcificaciones bilaterales y simétricas en el parénquima cerebral, con mayor compromiso de los núcleos de la base. ${ }^{7}$ Mientras que, el síndrome de Fahr se caracteriza por calcificaciones de los ganglios de la base secundarios a múltiples factores etiológicos como alteraciones bioquímicas, infecciosas, traumáticas, toxicas u otro trastorno sistémico. ${ }^{8}$

Dentro de las características clínicas de la enfermedad de Fahr y el síndrome de Fahr se observan las de origen neurológico como pérdida de la consciencia, convulsiones, 
Diferencias entre estas afecciones, según Perugula y Lippman. ${ }^{13}$

\begin{tabular}{|l|l|l|}
\hline & \multicolumn{1}{|c|}{ Enfermedad de Fahr } & \multicolumn{1}{c|}{ Síndrome de Fahr } \\
\hline Edad de inicio & 40 a 60 años & 30 a 40 años \\
\hline Rasgos genéticos & Autosómico dominante o recesivo & Ninguno \\
\hline Hallazgos radiológicos & $\begin{array}{l}\text { Calcificaciones estriato - pálido - dentadas } \\
\text { gruesas, progresivas, bilaterales y simétricas }\end{array}$ & $\begin{array}{l}\text { Calcificaciones intracraneales simétricas y } \\
\text { bilaterales }\end{array}$ \\
\hline Condiciones asociadas & Ninguna & $\begin{array}{l}\text { Endocrinopatías; anomalías bioquímicas; } \\
\text { causas infecciosas, traumáticas, toxicas u otro } \\
\text { trastorno sistémico }\end{array}$ \\
\hline Tratamiento & $\begin{array}{l}\text { Sin remedio especifico, solo tratamiento } \\
\text { sintomático }\end{array}$ & Tratamiento dirigido a la etiología especifica \\
\hline
\end{tabular}

trastorno del movimiento, trastorno de la marcha, miclonías, coreotetosis paroxística, fatigabilidad, movimientos involuntarios, calambres musculares, cambios sensoriales, anomalías cerebelosas: vértigos; $y$, las de origen psiquiátrico: deterioro cognitivo: demencia, delirio, confusión; síntomas psicóticos (alucinaciones, delirio, catatonia), irritabilidad, agresión, trastorno de personalidad, desorden de ánimo, ansiedad, comportamiento obsesivo. 9 En particular, es necesario destacar, un deterioro cognitivo progresivo de patrón subcortical. ${ }^{10}$

Los criterios de diagnóstico de la enfermedad de Fahr y del síndrome de Fahr se han modificado y derivado de Moskowitz, y se pueden expresar de la siguiente manera: 11,12

- Calcificación bilateral de los ganglios basales visualizada en neuroimagen.

- Disfunción neurológica progresiva, que generalmente incluye un trastorno del movimiento y/o manifestaciones neuropsiquiátricas.

- La edad de aparición es típicamente en la cuarta o quinta década de la vida.

- Ausencia de anomalías bioquímicas y características somáticas que sugieran una enfermedad mitocondrial o metabólica u otro trastorno sistémico.

- Ausencia de causa infecciosa, tóxica o traumática.

- Antecedentes familiares compatibles con herencia autosómica dominante.

Por lo general, en la literatura, el término enfermedad de Fahr y síndrome de Fahr se usan indistintamente, pero para las calcificaciones primarias de los ganglios basales sin etiología conocida, se debe utilizar el término enfermedad de Fahr; y, para las causas secundarias de calcificaciones de los ganglios basales con causas conocidas se debe utilizar el término síndrome de Fahr. En esta paciente, con calcificaciones cerebrales simétricas, sin etiología conocida, aunque no hay antecedentes genéticos, podríamos considerarla como un caso de enfermedad de Fahr.

Los autores consideran este caso de interés clínico debido a la correcta denominación de las patologías; y de la escasa información reportada que hay sobre este tema en nuestro medio.

\section{REFERENCIAS BIBLIOGRÁFICAS}

I. Bamberger H. Fahr disease, diagnosis in CT. J Neurol Neurosurg Psychiatry. 1855;45:67-9.

2. Fahr T. Idiopathische Verkalkung der Hirnge-fásse. Zentralblatt für allgemeine Pathologie und pathologische Anatomie. 1930; 50:129-33.

3. Gomille T, Meyer RA, Falkai P, Gaebel W, Konigshausen T, Christ F Prevalence and clinical significance of computerized tomography verified idiopathic calcinosis of the basal ganglia. Radiologe. 200 ; 4I: 205-10.

4. Pamo O. Chumpa R. Rosales K. Cabellos D. Enfermedad de Fahr. Revista de la Sociedad Peruana de Medicina Interna. 2013;26(I):50. URL disponible en: http://revistamedicinainterna.net/index.php/ spmi/issue/view/3।

5. Lira D. Custodio N. Montesinos R.Torre H. Calcificaciones cerebrales extensas y corea, en hipoparatiroidismo probablemente posquirúrgico. Anales de la Facultad de Medicina. 2009;70(2): I 19-122. URL disponible en: DOI: 10.1538I/anales.v70i2.960

6. Asokan AG, D'souza S, Jeganathan J, S. Fahr: una presentación de caso interesante.J Clin Diagn Res. 2013; 7 (3): 5302-5303. Disponible en: https://www.ncbi.nlm.nih.gov/pmc/articles/PMC5022990/

7. Saleem S, Aslam HM, Anwar M, Anwar S, Saleem M, SaleemA, et al. Fahr's syndrome: literature review of current evidence. Orphanet J Rare Dis. 2013;8:156.

8. Manyam BV. What is and what is not 'Fahr's disease'. Parkinsonism Relat Disord. 2005; I I:73-80.

9. Pistacchi M, Gioulis M, Sanson F, Marsala SM. Síndrome de Fahr y correlación clínica: serie de casos y revisión de la literatura. Folia Neuropathol. 2016; 54: 282-294.

10. Lauterbach EC, Cummings JL, Duffy J, Coffey CE, Kaufer D, Lovell M, Malloy P, Reeve A, Royall DR, Rummans TA, Salloway SP. Neuropsychiatric correlates and treatment of lenticulostriatal diseases: a review of the literature and overview of research opportunities in Huntingtons, Wilsons and Fahrs diseases. A report of the ANPA Committee on Research. American Neuropsychiatric Association.J Neuropsychiatry Clin Neurosci. 1998; 10: 249-66.

II. Moskowitz MA,Winickoff RN, Heinz ER. Calcificación familiar de los ganglios basales: un estudio metabólico y genético. N Engl J Med. | $97 \mid$ |; 285 (2): 72-77. doi: I0. 1056 / NEJMI $97 \mid 07082850202$.

12. Manyam BV. ¿Qué es y qué no es la enfermedad de Fahr? Parkinsonism RelatDisord.2005; I I (2):73-80.doi: I0.1016/j.parkreldis.2004.12.00I.

13. Perugula ML, Lippmann S. ¿Enfermedad de Fahr o síndrome de Fahr? Innov Clin Neurosci. 2016; 13: 45-46. URL disponible en: http://www. ncbi.nlm.nih.gov/pmc/articles/PMC5022990/

\section{CORRESPONDENCIA}

Carlos Alberto Dávila-Hernández

dh39130@hotmail.com

Fecha de recepción: |4-| | -2020.

Fecha de aceptación: |0-0|-202I. 